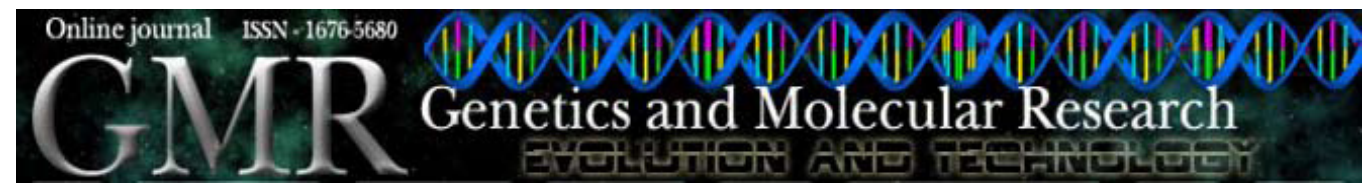

\title{
Genes up- and down-regulated by dermcidin in breast cancer: a microarray analysis
}

\author{
D.F. Moreira ${ }^{1}$, B.E. Strauss ${ }^{2}$, E. Vannier $^{3}$ and J.E. Belizário ${ }^{1}$ \\ ${ }^{1}$ Departamento de Farmacologia, Intituto de Ciências Biomédicas, \\ Universidade de São Paulo, São Paulo, SP, Brasil \\ ${ }^{2}$ Laboratório de Genética Molecular e Cardiologia/LIM-13, \\ Faculdade de Medicina, Universidade de São Paulo, São Paulo, SP, Brasil \\ ${ }^{3}$ New England Medical Center, Tufts University, Boston, MA, USA \\ Corresponding author: J.E. Belizário \\ E-mail: jebeliza@usp.br
}

Genet. Mol. Res. 7 (3): 925-932 (2008)

Received June 2, 2008

Accepted August 11, 2008

Published September 30, 2008

\begin{abstract}
Dermcidin (DCD) is a human gene mapped to chromosome 12 q13 region, which is co-amplified with multiple oncogenes with a well-established role in the growth, survival and progression of breast cancers. Here, we present a summary of a DNA microarray-based study that identified the genes that are up- and down-regulated in a human MDA-361 pLKO control clone and three clones expressing short hairpin RNA against three different regions of DCD mRNA. A list of 235 genes was differentially expressed among independent clones ( $>3$-fold change and $\mathrm{P}<0.005)$. The gene expression of 208 was reduced and of 27 was increased in the three DCD-RNAi clones compared to pLKO control clone. The expression of 77 genes (37\%) encoding for enzymes involved in amino acid metabolism, glucose metabolism and oxidoreductase activity and several genes required for cell survival and DNA repair were decreased. The expression of EGFR/ErbB-1 gene, an important predictor of outcome in breast cancer, was reduced together with the genes for betacellulin and amphiregulin, two known ligands of EGFR/ErbB
\end{abstract}


receptors. Many of the 27 genes up-regulated by DCD-RNAi expression have not yet been fully characterized; among those with known function, we identified the calcium-calmodulin-dependent protein kinase-II delta and calcineurin A alpha. We compared 132 up-regulated and 12 down-regulated genes in our dataset with those genes up- and downregulated by inhibitors targeting various signaling pathway components. The analysis showed that the genes in the DCD pathway are aligned with those functionally influenced by the drugs sirolimus, LY-294002 and wortmannin. Therefore, DCD may exert its function by activating the PI3K/AKT/mTOR signaling pathway. Together, these bioinformatic approaches suggest the involvement of DCD in the regulation of genes for breast cancer cell metabolism, proliferation and survival.

Key words: Dermcidin; Oncogene; Breast cancer; ErbB receptors

\section{INTRODUCTION}

Dermcidin (DCD) is a human gene normally expressed in the skin and brain (Schittek et al., 2001; Porter et al., 2003). The production of DCD protein and derived peptides increases in response to chemical stress, benign to malignant cell transition and infection by pathogenic microbes, suggesting it has a role in innate and immune surveillance (Hipfel et al., 2000; Schittek et al., 2001; Cunningham et al., 2002; Porter et al., 2003; Deans et al., 2006). We detected DCD and a splice variant (DCD-SV) in normal breast tissue, breast carcinoma cell lines and a subset of breast carcinoma samples from patients (Markovic J, Porter D, Moura R, Kashiabara J, et al., unpublished results). Transcripts of both genes were also detected in human skin, testis, placenta, and several tumor cell lines. Recombinant human DCD is a dimeric protein formed by two 11-kDa monomer that exhibit an unusually high susceptibility to proteolytic attack by trypsin (Majczak et al., 2007). The structural analysis of DCD protein in its native state revealed that it contains a poorly defined secondary structure that acquires $\alpha$-helix and $\beta$-sheet secondary structures in the presence of TFE/water mixtures and micellar SDS molecules (Majczak et al., 2007). Although DCD is expressed in a number of tissues, its functional role is unclear.

We have previously identified DCD as a highly expressed gene in the breast cancer cell line MDA-361 (Markovic J, Porter D, Moura R, Kashiabara J, et al., unpublished results). This is a luminal subtype of a breast cancer cell line derived from a brain metastasis with a high level of amplification of ERRB2, and positive expression for estrogen receptor and negative expression for progesterone receptor and tumor suppressor p53 (Neve et al., 2006). We have further explored DCD function and its contribution to breast cancer pathology by specifically knocking down its expression in MDA-361 cells using siRNA (Markovic J, Porter D, Moura R, Kashiabara J, et al., unpublished results). The polymerase chain reaction (PCR) and Western blot assays confirmed that DCD transcripts and protein levels were reduced in the three MDA clones expressing siRNA to different regions of its nucleotide sequence. We experimentally validated these results through functional studies in cell culture and an animal model (Moreira, 2007; Markovic J, Porter D, Moura R, Kashiabara J, et al., unpublished results). These studies showed that 
the knockdown of DCD increased the sensitivity of MDA-361 cells to cytotoxic drugs, confirming that DCD induces drug resistance in breast cancer cells as indicated in previous studies (Porter et al., 2003). Here, we present and examine a set of genes from the DNA-microarray gene expression profiles aimed at identifying the genes up- and downregulated in MDA-361 DCD-RNAi clones that correlate with the dermcidin gene response for this survival advantage.

\section{MATERIAL AND METHODS}

\section{Cell culture and characterization of DCD-RNAi clones}

Human MDA-361 cells were obtained from the American Type Culture Collection and maintained in DMEM supplemented with $4 \mathrm{mM} \mathrm{L}$-glutamine, $20 \%$ fetal bovine serum and $100 \mathrm{U} / \mathrm{mL}$ ampicillin and $100 \mathrm{mg} / \mathrm{mL}$ kanamycin sulfate, in a humidified atmosphere of $5 \% \mathrm{CO}_{2}$ in air at $37^{\circ} \mathrm{C}$. We have generated stable clones expressing DCD-RNAi for three regions of its mRNA and named them IBC-I (sense 5'-CCG GTC CTA GAT CCC AAG ATC TCC AAC TCG AGT TGG AGA TCT TGG GAT CTA GGT TTT TG-3' and anti-sense 5'-AAT TCA AAA ACC TAG ATC CCA AGA TCT CCA ACT CGA GTT GGA GAT CTT GGG ATC TAG GA-3'), IBC-II (sense 5'-CCG GTG GTT AGC CAG ACA GGC ACC AAC TCG AGT TGG TGC CTG TCT GGC TAA CCT TTT TG-3' and antisense 5'-AAT TCA AAA AGG TTA GCC AGA CAG GCA CCA ACT CGA GTT GGT GCC TGT CTG GCT AAC CA-3'), IBC-III (sense 5'-CCG GTA GCT GTA AGG AGA AGC TGA GAC TGC AGT CTC AGC CTC AGC TTC TCC TTA CAG CTT TTT TG3' and anti-sense 5'-AAT TCA AAA AAG CTG TAA GGA GAA GCT GAG ACT CGA GTC TCA GCT TCT CCT TAC AGC TA-3') and pLKO (vector control). These stable clones were cultured in the presence of puromycin $(2 \mu \mathrm{g} / \mathrm{mL})$ and routinely evaluated for DCD mRNA expression using real-time PCR and protein levels by Western blotting using a rabbit antibody raised against the DCD peptide sequence RQAPKPRKRSS (Porter et al., 2003). The expression of mRNA and protein is usually reduced $50-80 \%$ in DCDRNAi clones. To evaluate the biological effectiveness of DCD knockdown, we estimated cell survival rate by treating MDA-361 pLKO vector control and MDA-361 DCD-RNAi clones with $\mathrm{H}_{2} \mathrm{O}_{2}(0.0025 \%)$, staurosporine $(0.5 \mu \mathrm{M})$ and TNF $(200 \mathrm{ng} / \mathrm{mL})$ plus cyclohexamide $(5 \mu \mathrm{g} / \mathrm{mL})$ as described elsewhere (Markovic J, Porter D, Moura R, Kashiabara $\mathrm{J}$, et al., unpublished results). Usually, the cell survival rates decreased $20-50 \%$ in DCDRNAi clones, as compared to the MDA pLKO control clone.

\section{cRNA synthesis and labeling}

Total RNA was isolated from cells using the Qiagen's RNeasy Total RNA isolation kit and converted to double-stranded cDNA (ds-cDNA) in the presence of T7 promotertailed oligo-dT primer. The ds-cDNA was used as the template to produce the biotinylated complementary RNA (cRNA) in an in vitro transcription reaction catalyzed by T7 polymerase and containing biotinylated CTP and UTP in addition to the four unmodified ribonucleoside triphosphates. The cRNA was quantified spectrophotometrically, purified and chemically fragmented at $95^{\circ} \mathrm{C}$ for $35 \mathrm{~min}$. 


\section{Gene expression profiling}

RNA expression profiling was done using Affymetrix U133 Plus 2.0 human oligonucleotide microarrays. This microarray contains over 14,000 transcripts and variants from human genes. Hybridizations, washes and detection were carried out as recommended by the supplier. Briefly, the fragmented cRNA was added to a hybridization solution containing several biotinylated control oligonucleotides (for quality control), and hybridized to a microarray chip overnight at $45^{\circ} \mathrm{C}$. The bound cRNA was then fluorescently labeled using phycoerythrinconjugated streptavidin. Double signal amplification was performed with biotinylated antistreptavidin antibody with goat-IgG blocking antibody. Signal detection was performed with an Affymetrix GeneArray scanner and quantification with the Affymetrix GCOS software.

\section{Microarray data analysis}

DNA microarray data were analyzed by the DNA-Chip software (Li and Wong 2003). The expressions were compared between control MDA-361-pLKO clone and MDA-361-DCD-RNAi clones IBC-I, II and III. We considered for the analysis a Pearson's correlation coefficient $>95 \%$ and statistical significance of $\mathrm{P}<0.05$. The analyses were restricted to the set of genes with 3-fold changes in the gene expression organized in the functional categories in all braches of the hierarchical clustering tree (Whetzel et al., 2006). Network/pathway reconstruction from microarray data was analyzed using the GEST software (Hunter et al., 2001).

\section{RESULTS}

We used DNA oligonucleotide microarrays for human genome U133 Plus 2 manufactured by Affymetrix to identify genes in the MDA-361 breast carcinoma cell line that are differentially expressed between pLKO vector control clone and DCD-RNAi clones ICB-I, II, and III. A total of 235 genes were differentially and repeatedly expressed in the three independent clones analyzed in this study. Relative to pLKO vector control data profiling, 208 genes were up-regulated and 27 genes were down-regulated (3-fold change and statistical significance of $\mathrm{P}<0.05)$. Of 208 down-regulated genes, we identified 77 genes $(37 \%)$ encoding for enzymes involved in amino acid metabolism, glucose metabolism and oxidoreductase activity. Figure 1 shows the percentages according to their functional ontology. The levels of expression of several other genes involved in cell growth, DNA repair, stress oxidative response, and cell survival were decreased in the three DCDRNAi clones compared to control pLKO clone. Table 1 shows a list of the most important genes in these categories. DCD-RNAi silencing reduced the expression of the epidermal growth factor receptor (EGFR)/ErbB-1 and betacellulin and amphiregulin, two growth factors that bind to EGFR/ErbB-1 receptor (Table 1). The gene encoding vascular endothelial growth factor (VEGF) was also decreased. Among the 27 up-regulated genes in DCD-RNAi clones, we identified the genes for the catalytic subunit of calcineurin A and calcium/calmodulin-dependent protein kinase II delta (Table 1). Calcineurin (also called protein phosphatase-2B) is a calmodulin-regulated protein phosphatase, which plays an important role in signal transduction. 


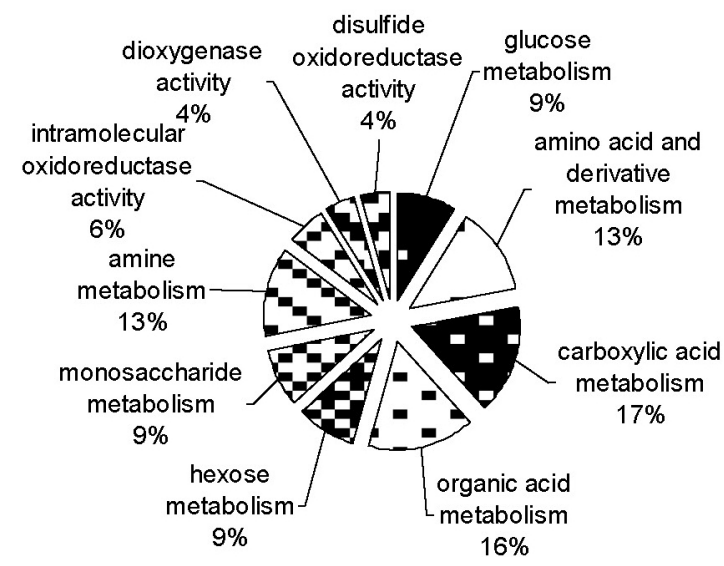

Figure 1. Functional grouping of genes down-regulated in DCD-RNAi clones compared to DCD-pLKO clone according to their functional ontology. cRNA were generated from ds-cDNA, hybridized in Affymetrix array chips (G-U133 Plus 2) and analyzed using the DNA-Chip software.

Table 1. Genes differentially expressed between MDA-361 breast carcinoma control pLKO clone and DCDRNAi IBC I, II and III clones ${ }^{1}$.

\begin{tabular}{|c|c|c|c|c|}
\hline $\begin{array}{l}\text { Accession } \\
\text { Affymetrix }\end{array}$ & Gene and function & $\begin{array}{l}\text { pLKO } \\
\text { clone }\end{array}$ & $\begin{array}{c}\text { IBC } \\
\text { clones }^{2}\end{array}$ & $\begin{array}{c}\text { Fold } \\
\text { change }\end{array}$ \\
\hline \multicolumn{5}{|c|}{ Genes down-regulated } \\
\hline \multicolumn{5}{|c|}{ Receptor and growth factors } \\
\hline 201983_s_at & Epidermal growth factor receptor (EGFR/ErbB-1) & 529 & 127 & -4.16 \\
\hline 205239_at & Amphiregulin (AREG) & 3774 & 781 & -5.09 \\
\hline 212171_x_at & Vascular endothelial growth factor (VEGF) & 1368 & 244 & -5.60 \\
\hline 241412_at & Betacellulin (BTC) & 213 & 46 & -4.60 \\
\hline \multicolumn{5}{|c|}{ Oxidative stress responce } \\
\hline 208658_at & Protein disulfide isomerase-associated 4 (P 4HB) & 1093 & 393 & -2.78 \\
\hline 202887_s_at & DNA-damage-inducible transcript 4 (DDIT 4) & 3994 & 683 & -5.84 \\
\hline 1554452_a_at & Hypoxia-inducible protein 2 (HIG2) & 2036 & 929 & -3.05 \\
\hline 211936_at & Heat shock 60-kDa protein (HSP A5) & 6583 & 1523 & -4.32 \\
\hline 1553972_a_at & Cystathionline beta-synthase (CBS) & 748 & 231 & -3.24 \\
\hline 202557_at & Stress 70 protein chaperone $(\mathrm{STCH})$ & 339 & 135 & -2.50 \\
\hline 202842_s_at & DnaJ (Hsp40) homolog (DNAJBI) & 1263 & 274 & -4.62 \\
\hline \multicolumn{5}{|c|}{ Genes up-regulated } \\
\hline \multicolumn{5}{|c|}{ Signal transduction } \\
\hline 236545_at & Protein phosphase 3, calcineurin A alpha (PPP3CA) & 276 & 1321 & +4.78 \\
\hline 231793_s_at & Calcium/calmodulin-dependent protein kinase (CAMK2D) & 247 & 1024 & +4.15 \\
\hline
\end{tabular}

${ }^{1}$ Microarray data were analyzed by the DNA-Chip software. The relative fold change in the expression of all genes in IBC DCD-RNAi clones was determined in relation to the expression in the control pLKO clone. ${ }^{2}$ Mean of the expression for combined data generated from IBC I, II and III clones.

In order to gain further insights into the underlying biological changes in an intracellular pathway, we compared 132 up-regulated and 12 down-regulated genes in our dataset (Figure 2) to the existing database containing the genes and their regulatory pathways influenced by the drugs emetine, strophanthidin, oubain, proscillaridin, sirolimus, LY-294002, and wortmannin, using the GEST software (Hunter et al., 2001). This analysis shows that DCD 
targeted a distinct group of genes generally elicited in cellular response to the drugs sirolimus $(\mathrm{P}=0.0002), \mathrm{LY}-294002(\mathrm{P}=0.0004)$ and wortmannin $(\mathrm{P}=0.0018)$. Therefore, it is likely that DCD exerts its functions by activating the PI3K/AKT/mTOR signaling pathway.

\section{DCD-RNAi \\ $\uparrow 12 \downarrow 132$ genes}

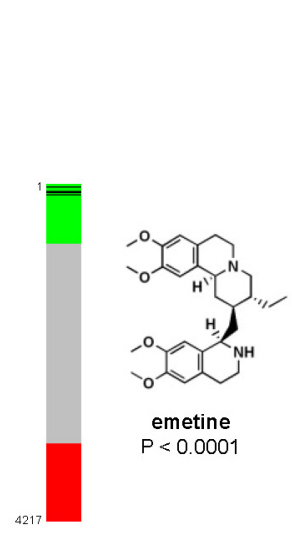

build 01.71 (4,217 instances; 1,269 discretes)

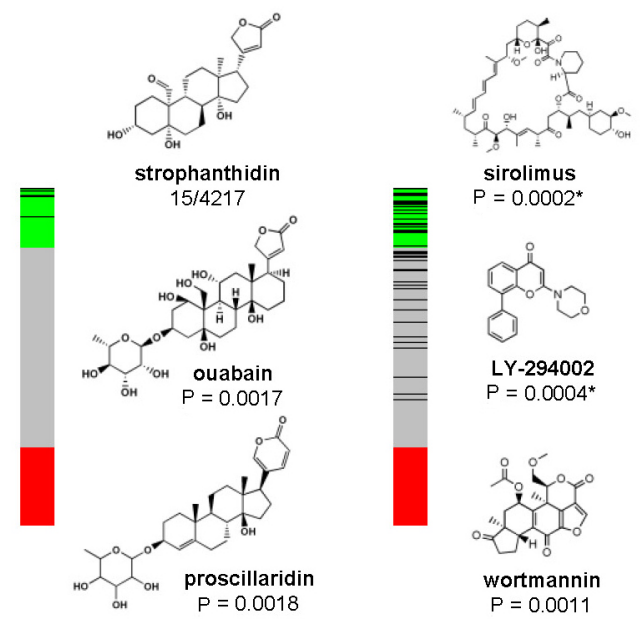

Figure 2. Each column represents an experiment and the black lines represent the genes in the DCD-RNAi dataset that were aligned with those identified during cellular responses to indicated drugs. The comparison shows that there is statistical correlation among genes related to the signal pathways influenced by the drugs sirolimus, LY294002 and wortmannin, which are specific inhibitors of mTOR, PI3K and AKT, respectively.

\section{DISCUSSION}

We have previously identified DCD as a highly expressed gene in a subset of breast cancer patients and various established breast cancer cell lines used as a model in breast cancer studies, including MDA-361, UACC 893, SUM185, and SUM 190 (Markovic J, Porter D, Moura R, Kashiabara J, et al., unpublished results). MDA-361 is a luminal subtype of a breast cancer cell line derived from a brain metastasis, which is characterized by an amplification of ErbB-2/HER/neu oncogene, positive expression for estrogen receptor and negative expression for progesterone receptor and tumor suppressor p53 (Neve et al., 2006). We have further explored DCD function and its contribution to breast cancer pathology by specifically knocking down its expression in MDA-361 breast cancer cell line using siRNA (Markovic J, Porter D, Moura R, Kashiabara J, et al., unpublished results). The PCR and Western blot assays confirmed that DCD transcripts and protein levels were reduced $(50-80 \%)$ in the three MDA clones expressing siRNA to different regions of its nucleotide sequence. We experimentally validated these results through functional studies in cell culture and in an animal model (Moreira, 2007; Markovic J, Porter D, Moura R, Kashiabara J, et al., unpublished results). These studies showed that the knockdown of DCD increased the sensitivity of MDA-361 cells to cy- 
totoxic drugs, confirming that DCD induces drug resistance in breast cancer cells as indicated in previous studies (Porter et al., 2003).

To identify the genes that are differentially up-regulated or down-regulated in response to DCD knockdown, we compared changes in mRNA levels between the pLKO vector control and three DCD-RNAi clones. A total of 235 genes within distinct functional categories were differentially expressed by at least a 3 -fold change. In this list, we identified a surprisingly higher number of genes for enzymes ( 77 genes) clustered in functional categories of the amino acid and glucose metabolism and intramolecular oxidoreductase activity (Figure 1). We also identified several genes coding for proteins involved in cell growth, survival and DNA repair mechanisms, among other genes (Table 1). The increased expression of enzymes involved in cellular metabolism is common in cells with higher oxidative and glycolytic activity such as cancer cells. Many of the 25 up-regulated genes in DCD-RNAi clones have not been fully characterized. Among those with known function, we identified two genes encoding signal transduction proteins (Table 1). The calcium-calmodulin-dependent protein kinase- and calcineurin-mediated signaling pathway is increased upon skeletal muscle differentiation (Xu et al., 2002). However, we did not identify genes linked to epithelial breast cell differentiation in the set of genes modulated by DCD in MDA-361 breast cancer cells.

EGFR/ErbB1/HER and the family members erbB2/HER/neu, ErbB3 (HER3) and erbB4 (HER4) and their ligands are involved in the development and progression of a variety of human cancers (Yarden and Sliwkowski, 2001). We noticed that the expression of EGFR/ErbB-1 and the ErbB receptor ligands betacellulin and amphiregulin were reduced after DCD knockdown in MDA-361 cells. The oncogene and co-receptor tyrosine kinase ErbB-2/HER/neu is genomically amplified in this breast cancer cell line (Neve et al., 2006). The expression of both EGFR/ErbB-1/ HER and ErbB-2/HER/neu is deregulated in $~ 30 \%$ of human breast cancers and associated with more aggressive disease and poor clinical outcome (Kauraniemi and Kallioniemi, 2006; Révillion et al., 2008). ErbB-2/HER/neu is the preferred dimerization partner for all the EGFR/ErbB-1 receptors. The activation of the ErbB network leads to receptor autophosphorylation and the recruitment of cytoplasmic signal transducers and transcription factors that regulate the proliferation, differentiation, motility, adhesion, and transformation of breast epithelial cells (Yarden and Sliwkowski, 2001). For this reason, it is likely that DCD expression may exert an active role in the EGFR/ErbBs signaling pathway during breast tumor initiation and maintenance.

We explored DCD gene function and association with biological pathways using the GEST software (Hunter et al., 2001). This analysis revealed that a significant number of genes upand down-regulated in DCD-RNAi clones are among these genes whose expression is influenced by the drugs LY-294002, wortmannin and sirolimus (Figure 2). These compounds exert their function by inhibiting the serine/threonine-specific kinases and protein phosphatase activity of proteins PI3K, AKT and mTOR, respectively. Activation of the PI3K/AKT signaling pathway stimulates cell growth, proliferation and survival via the phosphorylation of a number of AKT substrates, including the FOXO family transcription factors, mTOR, the eIF4E-binding proteins and the ribosomal p70-S6 protein kinase (Mamane et al., 2006). mTOR functions by integrating extracellular signals with amino acid availability and intracellular energy status to control translation rates and metabolic processes (Mamane et al., 2006). Collectively, these data support the hypothesis that DCD may influence cell growth and proliferation by activating the PI3K/AKT signal pathway.

In summary, DNA microarray data and functional and physiological studies, using MDA361 breast cancer cell line as a model, revealed that DCD coordinates the expression of genes 
involved in amino acid metabolism, glucose metabolism, oxidative stress, and cell survival. Based on these findings, we postulate that the up-regulation of genes for oxidative and survival responses is aimed at protecting cancer cells from toxic effects of reactive oxygen species generated in highly metabolic states, which is triggered by persisting proliferation activities of oncogenes and growth factors. Future in vivo studies aiming to biologically validate the genes and signaling pathways under DCD regulation may advance our understanding of its role in breast cancer pathophysiology.

\section{ACKNOWLEDGMENTS}

We thank Dr. Daniel Carrasco, Juan Yao, and Kornelia Polyak (Department of Medical Oncology, Dana-Farber Cancer Institute, Boston, MA, USA) for generously providing shRNAi constructs, DNA microarray data analysis, and constant collaboration throughout this project, and Priscilla Akamini for technical assistance. Research supported by FAPESP (\#2005/56909-0).

\section{REFERENCES}

Cunningham TJ, Jing H, Akerblom I, Morgan R, et al. (2002). Identification of the human cDNA for new survival/evasion peptide (DSEP): studies in vitro and in vivo of overexpression by neural cells. Exp. Neurol. 177: 32-39.

Deans DA, Wigmore SJ, Gilmour H, Tisdale MJ, et al. (2006). Expression of the proteolysis-inducing factor core peptide mRNA is upregulated in both tumour and adjacent normal tissue in gastro-oesophageal malignancy. Br. J. Cancer 94: 731-736.

Hipfel R, Schittek B, Bodingbauer Y and Garbe C (2000). Specifically regulated genes in malignant melanoma tissues identified by subtractive hybridization. Br. J. Cancer 82: 1149-1157.

Hunter L, Taylor RC, Leach SM and Simon R (2001). GEST: a gene expression search tool based on a novel Bayesian similarity metric. Bioinformatics 17 (Suppl 1): S115-S122.

Kauraniemi P and Kallioniemi A (2006). Activation of multiple cancer-associated genes at the ERBB2 amplicon in breast cancer. Endocr. Relat. Cancer 13: 39-49.

Li C and Wong WC (2003). DNA-Chip Analyzer (dChip). In: The Analysis of Gene Expression Data: Methods and Software (Parmigiani G, Garrett ES, Irizarry R and Zeger SL, eds.). Springer, New York, 120-141.

Majczak G, Lilla S, Garay-Malpartida M, Markovic J, et al. (2007). Prediction and biochemical characterization of intrinsic disorder in the structure of proteolysis-inducing factor/dermcidin. Genet. Mol. Res. 6: 1000-1011.

Mamane Y, Petroulakis E, LeBacquer O and Sonenberg N (2006). mTOR, translation initiation and cancer. Oncogene 25: 6416-6422.

Moreira DF (2007). Redução do crescimento e resistência celular de carcinoma mamário após silenciamento do gene PIF/ DCD via expressão de shRNAi. Master's thesis, Universidade de São Paulo, São Paulo.

Neve RM, Chin K, Fridlyand J, Yeh J, et al. (2006). A collection of breast cancer cell lines for the study of functionally distinct cancer subtypes. Cancer Cell 10: 515-527.

Porter D, Weremowicz S, Chin K, Seth P, et al. (2003). A neural survival factor is a candidate oncogene in breast cancer. Proc. Natl. Acad. Sci. U.S.A. 100: 10931-10936.

Révillion F, Lhotellier V, Hornez L, Bonneterre J, et al. (2008). ErbB/HER ligands in human breast cancer, and relationships with their receptors, the bio-pathological features and prognosis. Ann. Oncol. 19: 73-80.

Schittek B, Hipfel R, Sauer B, Bauer J, et al. (2001). Dermcidin: a novel human antibiotic peptide secreted by sweat glands. Nat. Immunol. 2: 1133-1137.

Whetzel PL, Parkinson H and Stoeckert CJ Jr (2006). Using ontologies to annotate microarray experiments. Methods Enzymol. 411: 325-339.

Xu Q, Yu L, Liu L, Cheung CF, et al. (2002). p38 Mitogen-activated protein kinase-, calcium-calmodulin-dependent protein kinase-, and calcineurin-mediated signaling pathways transcriptionally regulate myogenin expression. Mol. Biol. Cell 13: 1940-1952.

Yarden Y and Sliwkowski MX (2001). Untangling the ErbB signalling network. Nat. Rev. Mol. Cell Biol. 2: 127-137. 